\title{
artigo
}

Pessoa, J.D.; Gaudêncio, J.F.F.S.; Gonçalves, K.K.N.; Souza, B.F.N.; Castro, M.C.A.; Oliveira, T.L.B.;

Avaliação do perfil sociodemográfico e clínico de pacientes candidatos à cirurgia bariátrica

\section{Avaliação do perfil sociodemográfico e clínico de pacientes candidatos à cirurgia bariátrica}

Evaluation of the social demographic and clinical profile of patients candidate for bariatric surgery

Evaluación del perfil social demográfico y clínico de pacientes candidatos a cirugía bariátrica

\begin{abstract}
RESUMO
Objetivo: avaliar o perfil sociodemográfico e clínico de pacientes candidatos à cirurgia bariátrica. Método: estudo descritivo e transversal, realizado com candidatos ao processo de cirurgia bariátrica em hospital do Recife, Pernambuco, por meio de entrevista individual. A amostra foi composta por 120 indivíduos. Aplicou-se estatística descritiva para análise dos dados. Resultados: o perfil encontrado foi de pacientes do sexo feminino (78,3\%), de cor branca $(56,7 \%)$, solteiros $(50,0 \%)$, com média de idade de 35,8 anos e peso de 126,6 quilogramas. 0 uso de medicamentos para emagrecimento antes da cirurgia ocorreu em $79,2 \%$ dos participantes e a realização de dieta para controle de peso em $98,3 \%$. Foi evidenciada pelo menos uma comorbidade em $89,1 \%$ dos pacientes, sendo as principais esteatose hepática, lombalgia e hipertensão arterial. Conclusão: a maioria dos pacientes possuía fatores de risco para a obesidade e buscaram a cirurgia por motivos de saúde, após a tentativa de métodos não cirúrgicos.
\end{abstract}

DESCRITORES: Cirurgia bariátrica; Obesidade; Enfermagem.

\section{ABSTRACT}

Objective: to evaluate the sociodemographic and clinical profile of patients who are candidates for bariatric surgery. Method: a descriptive and cross-sectional study, carried out with candidates to the bariatric surgery process in a hospital in Recife, Pernambuco, through individual interviews. The sample consisted of 120 individuals. Descriptive statistics was applied for data analysis. Results: the profile found was made up of female patients $(78.3 \%)$, white $(56.7 \%)$, single $(50.0 \%)$, with a mean age of 35.8 years and a weight of $126,6 \mathrm{~kg}$. The use of weight loss medications before surgery occurred in $79.2 \%$ of the participants and the use of a diet for weight control in $98.3 \%$. At least one comorbidity was observed in $89.1 \%$ of the patients, the main ones being hepatic steatosis, low back pain and arterial hypertension. Conclusion: most patients had risk factors for obesity and sought surgery for health reasons, after trying non-surgical methods.

DESCRIPTORS: Bariatric Surgery; Obesity; Nursing.

\section{RESUMEN}

Objetivo: evaluar el perfil sociodemográfico y clínico de los pacientes candidatos a cirugía bariátrica. Método: estudio descriptivo y transversal, realizado con candidatos al proceso de cirugía bariátrica en un hospital de Recife, Pernambuco, mediante entrevistas individuales. La muestra estuvo formada por 120 individuos. Se aplicó estadística descriptiva para el análisis de datos. Resultados: el perfil encontrado estuvo conformado por pacientes del sexo femenino (78,3\%), blancas $(56,7 \%)$, solteras $(50,0 \%)$, con una edad media de 35,8 años y un peso de 126,6 kg. El uso de medicamentos para adelgazar antes de la cirugía ocurrió en el 79,2\% de los participantes y el uso de una dieta para el control del peso en el 98,3\%. Se encontró al menos una comorbilidad en el $89,1 \%$ de los pacientes, siendo las principales esteatosis hepática, lumbalgia e hipertensión arterial. Conclusión: la mayoría de los pacientes presentaba factores de riesgo de obesidad y acudió a cirugía por motivos de salud, tras probar métodos no quirúrgicos.

DESCRIPTORES: Cirugía bariátrica; Obesidad; Enfermería.

RECEBIDO EM: 28/02/2021 APROVADO EM: 05/04/2021

\section{Jessica Dantas Pessoa}

Acadêmica de Enfermagem no Centro Universitário Maurício de Nassau. Recife, Pernambuco, Brasil. ORCID: 0000-0001-8376-7992

\section{Jéssica Fernanda Freire da Silva Gaudêncio}

Enfermeira, Residente em Saúde Coletiva do Instituto de Medicina Integral Professor Fernando Figueira - IMIP. Recife, Per-nambuco, Brasil.

ORCID: 0000-0003-3542-4658 


\section{Karyne Kirley Negromonte Gonçalves}

Enfermeira, Mestre em Enfermagem e Professora do Centro Universitário Maurício de Nassau. Recife, Pernambuco, Brasil. ORCID: 0000-0002-0205-4112

\section{Bruno Felipe Novaes de Souza}

Enfermeiro, Mestre em Enfermagem e Professor do Centro Universitário Maurício de Nassau. Recife, Pernambuco, Brasil. ORCID: 0000-0001-5738-3717

\section{Maria da Conceição Alexandre Castro}

Enfermeira, Mestre em Biologia Parasitária e Professora do Centro Universitário Maurício de Nassau. Recife, Pernambuco, Brasil. ORCID: 0000-0002-4908-4415

\section{Terezinha Lima Barbosa de Oliveira}

Enfermeira, Especialista em Enfermagem do Trabalho e Coordenadora de Enfermagem do Centro Universitário Maurício de Nassau. Recife, Pernambuco, Brasil.

ORCID: 0000-0001-6176-5793

\section{INTRODUÇÃO}

A obesidade, por sua alta incidência, é considerada como problema de saúde pública. Ela é classificada como uma doença que se desenvolve a partir do excesso de gordura corporal, podendo resultar de fatores metabólicos, genéticos, sociais, culturais e comportamentais ${ }^{1}$. Além disso, é causadora de desordem nutricional e com elevada morbiletalidade, podendo acometer todo o sistema corporal humano ${ }^{2}$.

O controle do peso traz inúmeros benefícios à saúde e é ideal para contribuir com a diminuição de doenças relacionadas ao sobrepeso ${ }^{3}$. Quando esse agravo nutricional não é alcançado naturalmente, as pessoas buscam por tratamentos clínicos e cirúrgicos, sendo este último mais procurado por ter maior e mais rápida eficácia nos resultados em relação ao tratamento clínico convencional ${ }^{2,4}$.

A indicação terapêutica da cirurgia bariátrica deve ser realizada com acompanhamento multidisciplinar, pois o sucesso do tratamento depende de mudanças no hábito de vida e equilíbrio emocionals. O método mais simples e de baixo custo, rápido e acessível para definir o tratamento é a avaliação das medidas antropométricas, que são utilizadas para analisar o estado nutricional ${ }^{6,7}$. Os indivíduos com Índice de Massa Corpórea $(\mathrm{IMC}) \geq 40 \mathrm{~kg} / \mathrm{m}^{2}$ e aqueles que apresentam IMC $\geq 35 \mathrm{~kg} / \mathrm{m}^{2}$ associado a doenças correlacionadas ao peso excessivo têm indicação para realizar a gastroplastia ${ }^{3}$.
Ela é classificada

como uma doença

que se desenvolve a

partir do excesso de

gordura corporal,

podendo resultar de

fatores metabólicos,

genéticos, sociais,

\section{culturais e}

\section{comportamentais.}

A cirurgia bariátrica é considerada eficaz para tratar pacientes com obesidade grave8. De acordo com a Agência Nacional de Saúde Suplementar (ANS), além do IMC, também é critério avaliado para realização do procedimento cirúrgico em planos privados de assistência à saúde, ter idade entre 18 e 65 anos, apresentar falha de tratamento clínico por no mínimo dois anos e possuir obesidade mórbida há mais de cinco anos. Também se faz necessário que não seja um paciente psiquiátrico descompensado com risco para suicídio e usuário de álcool ou drogas ilícitas nos últimos cinco anos?

Quando os meios convencionais não são suficientes para alcançar o resultado almejado, torna-se comum levar em consideração a cirurgia bariátrica. $\mathrm{O}$ processo traz mudanças drásticas nos hábitos comportamentais e sociais, deixando a autoestima do indivíduo vulnerável e trazendo grandes mudanças alimentares, por isso se faz necessário um acompanhamento pré e pós-operatório pela equipe de enfermagem ${ }^{10}$.

Diante do exposto, o presente estudo objetivou avaliar o perfil sociodemográfico e clínico de pacientes candidatos a submissão da cirurgia bariátrica, a fim de subsidiar intervenções para a prática avançada da enfermagem a esse público.

\section{MÉTODO}

Trata-se de um estudo descritivo, quantitativo, de corte transversal, realizado entre os meses de outubro e novembro de 2020 em um hospital particular do município do Recife, Pernambuco, Brasil. A escolha do serviço se deu pela representatividade local de atendimento multidisciplinar e demanda de pacientes em busca do procedimento em estudo. A população foi caracterizada por pacientes candidatos à cirurgia bariátrica, cujo processo amostral não probabilístico por conveniência contou com cálculo de amostra para população seja finita, resultando em 120 pa- 
Tabela 1 - Características sociodemográficas de pacientes candidatos à cirurgia bariátrica. Recife, Pernambuco, Brasil, 2020.

VARIÁVEIS

Sexo

Feminino

Masculino

Raça

Branco

Negro

Pardo

Escolaridade

Ensino Fundamental Completo

Ensino Médio Incompleto

Ensino Médio Completo

Ensino Superior Incompleto

Ensino Superior Completo

Estado Civil

Casado/União Estável

53,0

60,0

7,0

70,0

58,3

Empregado

41,0

34,2

Desempregado

Estudante

Aposentado

Moradia

Casa Própria

98,0

81,7

Alugada

22,0

Agregado Familiar

Familia

116,0

Sozinho

4,0

3,3

Total

120,0

100,0

Fonte: autores, 2020

Tabela 2 - Características clínicas de pacientes candidatos à cirurgia bariátrica. Recife, Pernambuco, Brasil, 2020.

VARIÁVEIS

N

$\%$

Grau de Obesidade

Obesidade Grau II

17,0

14,2

Obesidade Grau III

103,0

85,8

Uso de Medicamentos para emagrecer

Sim

Não

25,0

20,8 cientes, com erro amostral de $5 \%$ e nível de confiança de $90 \%$.

Os critérios de inclusão utilizados foram: pacientes com idade igual ou superior a 18 anos, de ambos os sexos, apresentando obesidade igual ou acima do grau II e aqueles com obesidade estabelecida com tratamento clínico prévio insatisfatório. Foram excluídos aqueles que possuíam quaisquer barreiras de comunicação que impedissem as entrevistas.

A coleta de dados ocorreu na admissão do paciente ao pré-operatório. O início do atendimento com a equipe multidisciplinar acontece por meio da consulta de enfermagem, onde o paciente é admitido e são avaliados os critérios para o pré-operatório da cirurgia bariátrica, tais como grau de obesidade, tratamento prévio e condição emocional.

As entrevistas aconteceram no consultório de enfermagem, de forma individual, sendo guiada por questionário semiestruturado de autoria dos pesquisadores. Os pacientes que aceitaram participar assinaram o Termo de Consentimento Livre e Esclarecido. O estudo cumpriu com os preceitos éticos normatizados pela Resolução 466/12, sendo aprovado pelo Comitê de Ética da Universidade de Pernambuco HUOC/PROCAPE sob o Parecer de número 4.337.541 e CAAE 37826620.1 .0000 .5192$.

Os dados foram tabulados no editor de planilhas da Microsoft Office Excel@ e transportados para o software Statistical Package for the Social Sciences (SPSS), versão 21.0, onde foram analisados pelos pesquisadores por meio de estatística descritiva simples com cálculo de frequências absolutas e relativas para as variáveis categóricas e medidas de posição e dispersão para as variáveis contínuas.

\section{RESULTADOS}

Participaram do estudo 120 pacientes com indicação clínica para serem admitidos no processo pré-operatório de cirurgia bariátrica. A Tabela 1 apresenta o perfil sociodemográfico dos indivíduos incluídos na pesquisa. 
Realização de dieta para o controle de peso

Sim

118,0

98,3

Não

Acompanhamento da dieta

Com nutricionista

Por conta própria

Prática de Atividade Física

Sim

40,0

Não

80,0

Consumo de Álcool

Sim

Não

73

60,8

Uso de Tabaco

Sim

6,0

5,0

Não

114,0

95,0

Total

120,0

100,0

Fonte: autores, 2020.

Tabela 3 - Condições clínicas pré-existentes de pacientes candidatos à cirurgia bariátrica. Recife, Pernambuco, Brasil, 2020.

VARIÁVEIS

\section{Motivos para realizar a cirurgia bariátrica}

Tentou outros procedimentos sem êxito

Tentou outros procedimentos sem êxito e necessidade de saúde

Tentou outros procedimentos sem êxito, necessidade de saúde e estética

Diabetes Mellitus

Sim

Não

Hipertensão Arterial Sistêmica

Sim

Não

Esteatose hepática

Sim

Não

Lombalgia

Sim

Não

Presença de Transtornos Alimentares

Sim, mas recuperado

$11,0 \quad 9,2$

Sim, ainda em tratamento

Não

$98,0 \quad 81,6$

A prevalência dos pacientes quanto ao sexo foi feminina $(78,3 \%)$ e da cor branca $(56,7 \%)$. Pode-se observar que a maior parte era solteira $(50,0 \%)$, com instrução educacional de Ensino Superior Completo $(39,2 \%)$ e empregada $(58,3 \%)$. Além disso, foi identificada $81,7 \%$ possuía casa própria e $96,7 \%$ habitava nessas casas com a família. A faixa etária média, para ambos os sexos, foi de 35,8 anos. A Tabela 2 traz o resumo dos dados clínicos encontrados nesta investigação.

Verificou-se que $85,8 \%$ dos pacientes possuíam obesidade grau III e que a maioria $(79,2 \%)$ já havia feito uso de medicamentos para emagrecer. Do total de entrevistados, mais de $98 \%$ realizava dieta para controle de peso, sendo a maioria $(87,3 \%)$ acompanhada por nutricionista. Quanto às condições diárias de vida, foi identificado que a maior parcela dos pacientes não praticava exercício físico $(66,7 \%)$ e não faziam uso de álcool $(60,8 \%)$ e tabaco (95,0\%). A Tabela 3 exibe informações acerca da situação de saúde pré-operatória dos pacientes entrevistados.

Foi possível identificar que a maioria dos pacientes $(75,8 \%)$ tentou outros procedimentos antes de buscar a intervenção cirúrgica e apresentam necessidade de saúde. A média de idade em que os pacientes iniciaram a obesidade foi 22,1 anos. Acrescido a isso, percebeu-se a presença de diversas patologias, como diabetes, hipertensão, esteatose hepática e lombalgia, além de transtornos alimentares, humor deprimido e ansiedade.

\section{DISCUSSÃO}

Os resultados apontaram para um perfil sociodemográfico e clínico de pacientes com boas condições de vida, porém não isentos de fatores de risco para a obesidade. Alguns dados do estudo, a exemplo do sexo, foram semelhantes a estudos feitos em outras regiões do Brasil ${ }^{3,5}$. Acredita-se que o fato do sexo feminino prevalecer na procura pelo emagrecimento pode estar associado à pressão imposta pela sociedade de que a mulher para ser considerada bonita precisa ter o corpo magro ${ }^{14}$. Dife- 


$\begin{array}{lcc}\text { Humor Deprimido } & & \\ \text { Sim } & 30,0 & 25,0 \\ \text { Não } & 90,0 & 75,0 \\ \text { Ansiedade } & & \\ \text { Sim } & 79,0 & 65,8 \\ \text { Não } & 41,0 & 34,2 \\ \text { Total } & 120,0 & 100,0 \\ \text { Fonte: autores, 2020. } & & \end{array}$

rente das mulheres, a procura pelo emagrecimento por parte dos homens tende a surgir quando há uma dificuldade na realização de atividades diárias ${ }^{3}$.

A prevalência da cor branca entre os participantes pode estar associada ao poder aquisitivo por parte desta população, o que facilita o acesso às unidades particulares de saúde, como a que foi feita a pesquisa ${ }^{16}$. Já com relação ao estado civil, esta investigação diverge de outro estudo, que apresenta a maioria dos candidatos à cirurgia bariátrica como casados $1^{6,17}$. Esse número pode estar relacionado ao fato de que pessoas obesas, por não conseguirem corresponder às expectativas da mídia quanto ao corpo perfeito, se sentem inseguras e consequentemente desenvolvem dificuldade em conseguir prazer na convivência social e na construção de relacionamentos ${ }^{18}$.

A cirurgia bariátrica pode proporcionar aos pacientes reduções nos índices de morbimortalidade, o que explica a grande procura pelo procedimento ${ }^{16}$, desvelado no principal motivo para escolha do procedimento nesta pesquisa. $\mathrm{O}$ peso médio encontrado foi de $126,6 \mathrm{~kg}$ e a altura de $1,65 \mathrm{~m}$. Assim, é possível inferir que o IMC apresentou média de 46,3 (obesidade de grau III), a qual já se encontra em nível muito alto de gordura corporal. Nessa condição, os pacientes já estão acometidos por outras comorbidades e são candidatos com grandes chances para o procedimento cirúrgico, levando em consideração que a bariátrica é o tratamento mais eficaz para obesidade grau III $^{16}$.

Os resultados mostraram que grande parte dos entrevistados possuíam sobrepeso em fases precoces da vida. Esse fator está associado ao risco do desenvolvimen- to e permanência da obesidade na fase adulta. Para a prevenção desta ocorrência, torna-se necessário que o enfermeiro invista em intervenções focadas na reeducação alimentar e incentivo de hábitos saudáveis durante o período escolar, assim, possivelmente os hábitos saudáveis aprendidos durante esta faixa etária serão transferidos para a idade adulta, diminuindo assim a chance de desenvolver a obesidade ${ }^{20}$.

\section{A cirurgia}

bariátrica pode proporcionar aos pacientes reduçôes nos índices de morbimortalidade, o que explica a grande procura pelo procedimento

Em pacientes candidatos à cirurgia bariátrica, o ideal é que o enfermeiro conte com o auxílio do nutricionista para acompanhamento da dieta, pois assim os indivíduos estarão mais preparados para aceitar a alimentação no período pós-operatório $^{23-24}$. Estudo aponta que o tratamento farmacológico ajuda o paciente a perder peso, mas a eficácia da medicação por tempo maior que dois anos não é estabelecida completamente ${ }^{15}$.

A prática de atividade física é um fundamental para a perda de peso e auxílio na melhoria da qualidade de vida. Pesquisa realizada na Clínica de Cirurgia da Obesidade e do Aparelho Digestivo de Santa Maria-RS divulgou que 69\% dos candidatos à cirurgia realizavam atividade física, isso pode ser explicado devido ao acompanhamento por equipe multidisciplinar no pré-operatório, que incentiva o paciente às práticas ${ }^{15}$.

Algumas comorbidades vêm sendo percebidas em pacientes sujeitos à cirurgia. De acordo com a literatura, a depressão é o transtorno psiquiátrico que mais se relaciona com a obesidade mórbida ${ }^{16}$. Assim como encontrado no presente estudo, a ansiedade também aparece em outras pesquisas, onde indivíduos obesos, principalmente do sexo feminino, possuem maiores chances de desenvolver transtornos ansiosos quando comparado a indivíduos com IMC dentro dos parâmetros considerados normais ${ }^{21}$. Autores afirmam que pacientes com obesidade podem utilizar os alimentos como um escape para preencher vazios emocionais $^{18}$.

Além de doenças emocionais, candidatos à cirurgia bariátrica apresentam doenças pré-existentes importantes para o direcionamento da assistência, por isso devem ser bem avaliados pelo enfermeiro ${ }^{3}$. Nesta investigação foram identificadas comorbidades relacionadas ao peso, como diabetes, dislipidemias, apneia obstrutiva do sono, doenças osteomusculares degenerativas, hipertensão arterial, esteatose hepática e lombalgia, sendo as três últimas relatadas com maior frequência, diferentemente de outros estudos, onde a hipertensão e diabetes apresentam resultados mais altos ${ }^{22}$.

\section{CONCLUSÃO}

Foi possível evidenciar que a maioria dos pacientes buscam realizar a cirurgia bariátrica por necessidades de saúde, após 
tentativas sem sucesso de métodos não cirúrgicos, uma vez que apresentam patologias físicas e psíquicas, além de apresentarem relações sociais prejudicadas. Muitos dos participantes do estudo iniciaram a obesidade ainda na infância e passaram a vida em tratamento clínico, realizando dieta e fazendo uso de medicações para redução de peso, porém, sem resultados favoráveis. A avaliação sociodemográfica e clínica de tais pacientes faz-se necessária, pois permite aos enfermeiros conhecer e traçar estratégias eficazes, mediante as principais necessidades do cliente cirúrgico no perioperatório, para que assim, não venham desenvolver complicações durante o procedimento cirúrgico ou no pós-operatório de cirurgia bariátrica.

\section{REFERÊNCIAS}

1. Oliveira LSF, Mazino Filho ML, Castro JBP, Touguinha HM, Silva PCR, Ferreira MEC. Repercussões da cirurgia bariátrica na qualidade de vida, no perfil bioquímico e na pressão arterial de pacientes com obesidade mórbida. Fisioterapia e pesquisa. 2018;25:284-293.

2. Silvério AO, Rebelo BRR, Prudente CA, Martins COM, Oliveira $\mathrm{PMC}$, Cardoso DMM, et al. Balão intragástrico como tratamento ponte para a cirurgia bariátrica. GED gastroenterol. endosc. dig. 2017; 36 (3):109-114.

3. Castanha CR, Ferraz AAB, TCBC-PE, Castanha AR, Belo GQMB, Lacerda RMR, et al. Avaliação da qualidade de vida, perda de peso e comorbidades de pacientes submetidos à cirurgia bariátrica. RevColBras Cir. 2018;45(3):1-9.

4. Querido JD, Aguiar LR, Justino MP, Faria SO, Silva RR, Grasselli CSM. Estado nutricional antropométrico e comorbidades associadas à síndrome metabólica em mulheres submetidas à cirurgia bariátrica. Sci Med. 2016 Outubro;26(3):1-7.

5. Rêgo AS, Zulin A, Scolari S, Marcon SS, Radovanovic CAT. Análise das condições clínicas de pessoas obesas em período pré e pós-operatório de cirurgia bariátrica. Rev. Col. Bras. Cir. 2017;44(2):171-178.

6. Medeiros KF, Silva ALS, Fernandes ACCF, Martins MCC. Composição corporal e avaliação antropométrica de adultos. Revenferm UFPE online. 2015;9(Supl. 10):1453-1460.

7. Fernandez M, Toimil RF, Rasslan Z, Ilias EJ, Gradinar ALT, Malheiros CA. Avaliação da gordura corporal em pacientes obesas no pré-operatório de cirurgia bariátrica. ABCD ArqBrasCirDig. 2016;29(Supl.1):59-61

8. Barros LM, Moreira RAN, Frota NM, Araújo TM, Caetano JA. Qualidade de vida entre obesos mórbidos e pacientes submetidos à cirurgia bariátrica. Rev.Eletr.Enf. 2015 Abr-Jun;17(2):312-21.

9. Cobertura: gastroplastia (cirurgia bariátrica) por videolaparoscopia ou via laparotômica. Agência Nacional de Saúde Suplementar. 2019 May 17;

10. Justino YAC, Tatagiba TMB, Pogian LP, Pimentel FC. Modificações comportamentais entre o pré e o pós-operatório de pacientes bariátricos. Psicologia em Revista. 2018 Agosto;24:577-599.

11. Elias AA, TCBC-SP, Oliveira MR, Campos JM, TCBC-PE, Sasake WT, et al. Cirurgia bariátrica robótico-assistida: análise de série de casos e comparação com via laparoscópica. RevColBras Cir. 2018;45(3):1-9.

12. Chaim EA, Pareja JC, Gestic MA, Utrini MP, Cazzo E. Preoperativemultidisciplinaryprogram for bariatricsurgery: a pro- posal for theBrazilianPublic Health System. ArqGastroenterol. 2017;54:70-74.

13. Barros LM, Moreira RAN, Frota NM, Caetano JA. Identificação dos diagnósticos de enfermagem da classe de respostas cardiovasculares/pulmonares em pacientes submetidos à cirurgia bariátrica. Aquichan. 2015 Junho;15:200-209.

14. Oliveira DM, Merighi MAB, Jesus MCP. A decisão da mulher obesa pela cirurgia bariátrica à luz da fenomenologia social. Rev Esc Enferm USP. 2014 Oct 14;48(6):970-976.

15. Silva PT, Patias LD, Alvarez GC, Kirsten VR, Colpo E, Moraes CMB. PERFIL DE PACIENTES QUE BUSCAM A CIRURGIA BARIÁTRICA. ABCD Arq Bras Cir Dig. 2015;28(4):270-273.

16. Araújo GB, Brito APSO, Mainardi CR, Martins Neto ES, Centeno DM, Brito MVH. Perfil clínico-epidemiológico de pacientes submetidos à cirurgia bariátrica. Araújo et al. Para Res Med. 2018;1(4):1-8.

17. Barros LM, Brandão MGSA, Ximenes MAM, Fontenele NAO, Caetano JA. Perfil clínico-epidemiológico de pacientes adultos em fila de espera para cirurgia bariátrica. Revista enfermagem atual in derme. 2019;88(26):1-9.

18.De Lima ACR, Oliveira AB. Fatores psicológicos da obesidade e alguns apontamentos sobre a terapia cognitivo-comportamental. Mudanças-Psicologia da Saúde. 2016;24(1):1-16.

19.Scherer A, Moré C, Motta C, Coradini A, De Farias R. Rede social significativa e de suporte social: impacto no tratamento bariátrico. Psicologia, Saúde \& Doenças. 2019;20(3):630-650.

20.Quesada K, Detregiachi CRP, Barbalho SM, Oliveira MRM, Rasera I, Vaz EC, Goulart RA. PERFIL SOCIOECONÔMICO E ANTROPOMÉTRICO DE CANDIDATAS À CIRURGIA BARIÁTRICA PELO SISTEMA ÚNICO DE SAÚDE. Revista Saúde e Pesquisa. 2015;8(3):431-438.

21.Andric JT, Méa CPD, Ferreira VRT, Vesco JAD, Vesco AIAD. Sintomas de ansiedade em pacientes pré-cirurgia bariátrica: um estudo comparativo. Contextos Clínicos. 2019;12:779-795.

22.Oliveira RMM, Passos XS, Marques MS. Perfil do indivíduo candidato à cirurgia bariátrica no Hospital Geral de Goiânia-GO. J Health Sci Inst. 2015;31(2):172-175.

23.Ades L, Kerbauy RR. Obesidade: realidades e indagações. Psicologia USP. 2002;13(1):197-216.

24. Silveira MS, Oliveira TM, Oliveira AS, Santos DTS, Menezes RR, Lima ML. Conhecimento nutricional entre mulheres obesas e eutróficas atendidas em uma unidade ambulatorial de saúde: classificação e comparação entre os grupos. SaudColetiv. 2020; 10(59):4298-313. 